\title{
Up-Regulated MicroRNA-27b Promotes Adipocyte Differentiation via Induction of Acyl-CoA Thioesterase 2 Expression
}

\author{
Yuka Murata, ${ }^{1}$ Takashi Yamashiro ${ }^{D},{ }^{1}$ Takaomi Kessoku, ${ }^{2}$ Israt Jahan, ${ }^{3}$ Haruki Usuda, ${ }^{3}$ \\ Tetsuya Tanaka, ${ }^{3}$ Takayuki Okamoto $\mathbb{D}^{\mathrm{D}},{ }^{3}$ Atsushi Nakajima $\mathbb{D D}^{2},{ }^{2}$ and Koichiro Wada ${ }^{3}{ }^{3}$ \\ ${ }^{1}$ Department of Orthodontics and Dentofacial Orthopedics, Graduate School of Dentistry, Osaka University, Suita 565-0871, Japan \\ ${ }^{2}$ Department of Gastroenterology and Hepatology, Yokohama City University School of Medicine, Yokohama 236-0004, Japan \\ ${ }^{3}$ Department of Pharmacology, Shimane University, Faculty of Medicine, Izumo 693-8501, Japan
}

Correspondence should be addressed to Takayuki Okamoto; okamoto@med.shimane-u.ac.jp and

Koichiro Wada; koiwada@med.shimane-u.ac.jp

Received 22 April 2019; Revised 22 July 2019; Accepted 4 August 2019; Published 17 December 2019

Academic Editor: Fabrizio Montecucco

Copyright (C) 2019 Yuka Murata et al. This is an open access article distributed under the Creative Commons Attribution License, which permits unrestricted use, distribution, and reproduction in any medium, provided the original work is properly cited.

\begin{abstract}
Nonalcoholic fatty liver disease (NAFLD) is characterized by a spectrum of liver pathologies, from simple steatosis to steatohepatitis. Recent studies have increasingly noted the aberrant expression of microRNAs closely related to NAFLD pathologies. We have previously shown the presence of increased levels of microRNA-27b (miR-27b) in patients with NAFLD. In this study, we investigated the role of miR-27b in NAFLD by examining the impact of up-regulated miR-27b on the differentiation of preadipocytes into mature adipocytes. We found that miR-27b-3p remarkably enhances the adipocyte differentiation of 3T3-L1 cells associated with lipid accumulation and intracellular triglyceride contents. Furthermore, we have demonstrated not only that miR-27b-3p induces acyl-CoA thioesterase 2 (ACOT2) expression in 3T3-L1 cells, but also that the knockdown of ACOT2 suppresses lipid accumulation and adipocyte differentiation in both the presence and absence of miR-27b-3p treatment. Our data strongly suggest that the miR27b-ACOT2 axis is an important pathway in adipocyte differentiation and may play a role in the pathogenesis of NAFLD.
\end{abstract}

\section{Introduction}

Nonalcoholic fatty liver disease (NAFLD), the most common cause of chronic liver disease, is characterized as a spectrum of liver disease ranging from simple steatosis to nonalcoholic steatohepatitis (NASH), which is the most severe form of NAFLD and involves significant hepatocellular injury, fibrosis, and inflammation [1]. NAFLD is associated with obesity, type 2 diabetes mellitus, hyperlipidemia, and insulin resistance, and is closely related to increased cardiovascular events and hepatic disease-related mortality [2]. Moreover, NASH frequently progresses to liver cirrhosis and hepatocellular carcinoma [3]. Adipocyte differentiation from preadipocyte is a prerequisite for the onset of steatosis and includes lipid accumulation and enlargement of liver tissue in the initial stages of NAFLD [4]. Thus, blockade of aberrant adipocyte differentiation leading to lipid deposition in liver tissue is recognized as an effective approach for preventing the development of NAFLD.

Recently, microRNAs (miRNAs) have been touted as a therapeutic target and specific biomarker not only for NAFLD, but also for several cancers and hepatitis C [5]. miRNAs are small (18-25 nucleotides) noncoding RNAs [6] that target the $3^{\prime}$-untranslated regions of their target mRNAs in order to regulate gene expression [7, 8]. miRNAs act in the posttranscriptional regulation of gene expression through inhibition of translation or mRNA degradation [8]. miRNAs are constitutively expressed as one group of regulators in a number of biologic processes [8]. Several studies have analyzed differential miRNA expression in patients with NAFLD and have identified a number of miRNAs at increased or decreased levels during the development of NAFLD and NASH $[5,9]$.

Previous studies have shown that miRNAs and their regulatory networks are implicated in the pathogenesis of NAFLD and NASH. Reduced expression of miR-122 was observed in hepatic tissues of NAFLD and NASH [9]. In contrast, miR-122 expression was elevated in the serum or blood of NAFLD patients [10]. Knockout of miR-122 expression facilitated triglycerides (TGs) accumulation and hepatic steatosis that progressed to NASH and fibrosis [11]. In addition, miR-34a was up-regulated in tissue and serum of 
NAFLD patients, and induced down-regulation of NAFLD related genes leading to TGs accumulation and steatosis [9]. Moreover, a number of miRNAs and their target genes has been identified in NAFLD and NASH patients suggesting that the dyscoordination of coding-noncoding RNA regulatory network is important trigger of NAFLD pathogenesis.

In our previous studies, we also compared alterations in miRNA expression in the liver biopsy samples of NASH patients against healthy subjects via extensive miRNA microarray analysis $[12,13]$. This result showed reduced expression of miR-122 in liver tissues of NAFLD and NASH patients as well as previous reports. Among altered miRNAs in patients, we found that miR-27b is remarkedly increased in liver tissue and serum from the early phase of NAFLD to severe NASH $[12,13]$. It has been reported that miR-27b is involved in cell proliferation, differentiation, migration, invasion, metastasis, multi-drug resistance in various tumor cells [14-18]. However, the exact role of miR-27b in NAFLD pathogenesis, and in particular its relationship to adipocyte differentiation, remains unclear.

In this study, we have investigated the role of miR-27b in 3T3-L1 cell differentiation into adipocytes and other potential mechanisms. We found that miR-27b functionally accelerated mature adipocyte differentiation and excessive lipid accumulation through the induction of acyl-CoA thioesterase 2 (ACOT2) expression.

\section{Materials and Methods}

2.1. Cell Culture and Induction of Adipocyte Differentiation. Mouse 3T3-L1 preadipocytes obtained from Primary Cell (Sapporo, Japan) are commonly used to study in vitro adipocyte differentiation. We employed 3T3-L1 cells and induced adipocyte differentiation using a previously described method [19, 20]. Briefly, 3T3-L1 cells were cultured in Dulbecco's modified Eagle's medium (DMEM, Sigma Aldrich, Tokyo, Japan) supplemented with $10 \%$ fetal bovine serum (Equitech-Bio, Taxas, USA), 1\% MEM nonessential amino acids (Nacalai tesque, Kyoto, Japan), $100 \mathrm{IU} / \mathrm{mL}$ penicillin and $0.1 \mathrm{mg} / \mathrm{mL}$ streptomycin (Nacalai tesque). Three days after reaching confluency (day 0 ), the medium was replaced with differentiation medium containing $150 \mathrm{nM}$ insulin (INS, Sigma Aldrich), $1 \mu \mathrm{M}$ dexamethasone (DEX, Sigma Aldrich), $100 \mu \mathrm{M}$ 3-Isobutyl-1-methylxanthine (IBMX, Sigma Aldrich) and $1 \mu \mathrm{M}$ Rosiglitazone (ROSI, GlaxoSmithKline, Tokyo, Japan). The differentiation medium was changed every 4 days until analysis (day 6).

\subsection{Evaluation of Lipid Accumulation and Adipocyte Differen-}

tiation. Lipid accumulation in adipocytes was visualized by Oil red $\mathrm{O}$ staining. Cells stained by Oil red $\mathrm{O}$ were observed via microscopy. The amount of triglyceride (TG), an index of lipid accumulation, was quantitatively measured by using a Triglyceride E-test Wako kit (Wako Pure Chemicals, Tokyo, Japan). The amount of TG was normalized by the protein amount.
2.3. Transfection of MiR-27b-3p into 3T3-L1 Cells. Synthesized mimic miR-27b-3p (mature miRNA sequence: AGA GCU UAG CUG AUU GGU GAA C) or negative control (miRNA mimic Negative control) was purchased from Life Technologies (Tokyo, Japan). Negative control or miR-27b-3p was transfected by Lipofectamine RNAiMAX transfection reagent (Life Technologies) into 3T3-L1 cells at a final concentration of $20 \mathrm{nM}$. Transfection of negative control or miR-27b-3p was performed at day 0 and day 3 , respectively. After transfection for 24 hours, transfection medium was replaced with differentiation medium. Adipocyte differentiation and lipid accumulation in cells were assessed at day 6 by Oil red $O$ staining and a TG assay. The cell number at day 6 was counted by using a Countess Automated Cell Counter (Thermo Fisher Scientific, Tokyo, Japan).

\subsection{DNA Microarray Analysis of MiR-27b-Transfected 3T3-L1}

Cells. After a second transfection of miR-27b-3p or negative control for 24 hours, total RNA was purified by using TRizol reagents (Thermo Fisher Scientific) and applied for KuraboDNA microarray analysis (KURABO Bio-Medical department, Osaka, Japan). The relative expression level of a given mRNA was calculated by comparing the signal intensities of valid spots throughout the microarray experiments. All analyzed data were scaled by global normalization and were expressed as the ratio of the signal of cells. We selected genes that showed more than a 1.7 -fold induction or less than a 0.6 -fold repression according to the previously described methods $[20,21]$. Gene ontology (GO) enrichment analysis of differentially expressed genes was assessed with PANTHER (http://www.pantherdb.org/) and then focused on genes in relation to adipocyte differentiation and lipid metabolisms by referring to the gene's function.

2.5. Detection of ACOT2 Protein Expression by Western Blotting. After transfection and differentiation, 3T3-L1 cells were harvested in lysis buffer with $0.1 \%$ of a proteinase inhibitor cocktail (Promega, Tokyo, Japan). Equal amounts of protein were subjected to SDS-PAGE and analyzed by Western blotting. ACOT2 protein was detected using anti-ACOT2 polyclonal antibody (Proteintech, Chicago, IL; 1:1000) and peroxidase-conjugated anti-rabbit IgG (Bio-Rad Laboratories, Richmond, CA; 1:2000). Glyceraldehyde-3-phosphate dehydrogenase (GAPDH) protein was detected using anti-GAPDH antibody (Trevigen, Gaithersburg, MD) and peroxidase-conjugated anti-rabbit IgG. Blots were developed by chemiluminescence (Immobilon Western, Millipore, Billerica, MA).

\subsection{Knockdown of ACOT2 Expression in 3T3-L1 Cells by RNA}

Interference. Specific small interfering RNAs (siRNAs) against ACOT2 mRNA were designed by Stealth RNAi-siRNA system (Life Technologies). The sense and antisense sequences of ACOT2-siRNA were $5^{\prime}$-UGG UGG CCU CGU CUU UCG CUG UCC U-3', and $5^{\prime}$-AGG ACA GCG AAA GAC GAG GCC ACC A-3', respectively. ACOT2-siRNAs or negative control siRNAs (Stealth RNAi Negative Control Duplexes) were transfected by Lipofectamine RNAiMAX at a final 


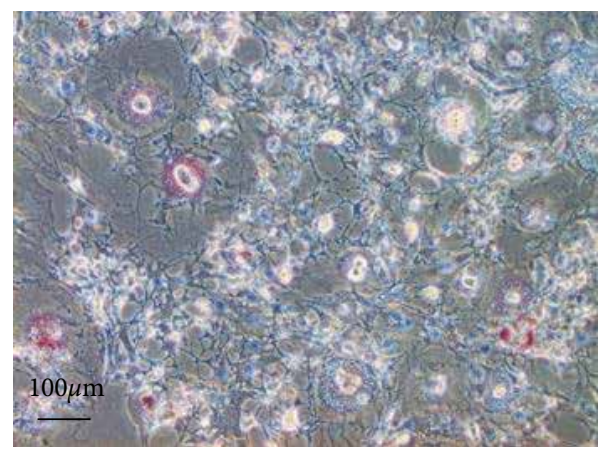

Control

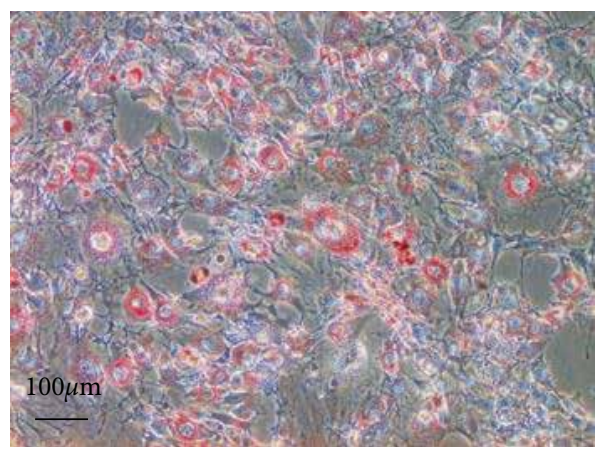

miR-27b-3p

(a)

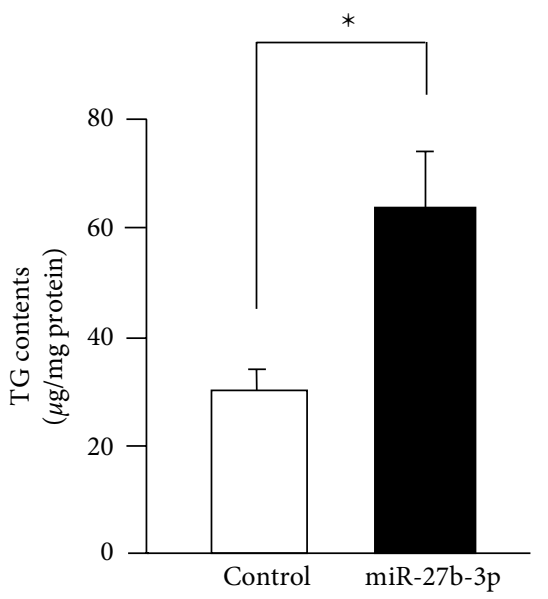

(b)

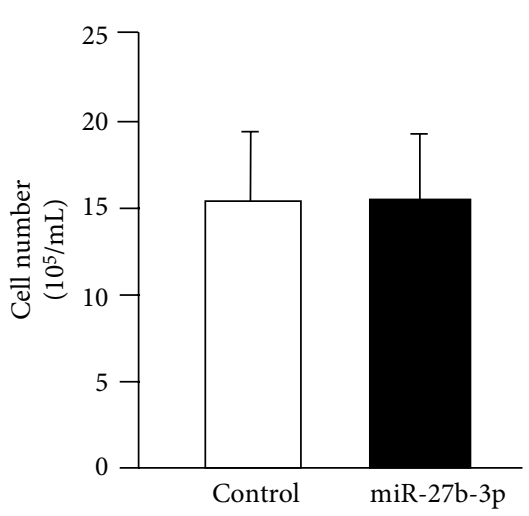

(c)

FIGURE 1: MiR-27b increases lipid accumulation and adipocyte differentiation. (a) Oil red O staining of 3T3-L1 cells after adipocyte differentiation. At day 0 and day 3, control-miR (left panel) and miR-27b (right panel) were transfected into 3T3-L1 cells. Representative data from three different images are shown. Scale bars: $100 \mu \mathrm{m}$. (b) Intracellularly TG contents of control-miR and miR-27b transfected 3T3-L1 cells were measured. Each column represents the mean \pm SEM from 6 independent experiments. ${ }^{*}$ Significantly difference than control-miR by Student's $t$-test $(P<0.05)$. (c) Numbers of control-miR or miR-27b-transfected 3T3-L1 cells after adipocyte differentiation. Each column represents the mean \pm SEM from 3 independent experiments.

concentration of $10 \mathrm{nM}$ siRNA. After transfection for 24 hours, the medium was replaced with differentiation medium to induce differentiation. Transfection of siRNAs was performed at day 0 and day 3 . As combination treatment with miR-27b-3p and ACOT2-siRNA, both transfection-reagents, was applied to cultured cells at the same time. After differentiation, lipid accumulation and cell numbers were evaluated.

2.7. Statistical Analysis. Results are expressed as the mean \pm SEM. Statistical comparisons of TG contents and cell proliferation between control-miR and miR-27b-3p were performed using the Student's $t$-test. Statistical comparisons of TG contents between control-miR, miR-27b-3p, and combination with miR-27b-3p and ACOT siRNA were performed using Scheffe's method after analysis of variance (ANOVA). The results were considered significantly different at $P<0.05$.

\section{Results}

3.1. MiR-27b-3p Increased Lipid Accumulation and TG Contents in 3T3-L1 Cells after Adipocyte Differentiation. Adipocyte differentiation is primarily controlled by CCAAT/enhancer binding protein (C/EBP) and PPAR $\gamma$ at the transcriptional level $[22,23]$. In the experimental model, DEX, IBMX, INS, and ROSI were used as inducing agents of adipocyte differentiation. We confirmed adipocyte differentiation after these stimulation processes (data not shown). In order to examine the effect of miR-27b on adipocyte differentiation, we transfected mimetic miR-27b-3p into 3T3-L1 cells and evaluated lipid deposition in the intracellular space following differentiation. Transfection of miR-27b-3p remarkedly increased lipid deposition in 3T3L1 cells compared to transfection of control-miR (Figure 1(a)). TG contents, which represent the index of mature adipocyte, were also significantly higher in miR-27b-3p-transfected cells, more so than in control-miR-transfected cells. To investigate the effect of miR-27b on cell proliferation, we determined the number of miR-27b-3p-transfected cells after differentiation (Figure 1(b)). In contrast to control-miR-transfected cells, miR-27b-3p did not influence the total cell number during our experimental period and conditions (Figure 1(c)). These results, which show that miR-27b enhanced adipocyte 
TABLE 1: Microarray analysis of gene expression increases on miR-27b transfection.

\begin{tabular}{|c|c|c|}
\hline Gene description & Gene accession & Fold increase \\
\hline Prolactin family 2 , subfamily c, member $5(\operatorname{Prl} 2 \mathrm{c} 5)$ & NM_181852 & 3.672185 \\
\hline Serum amyloid A 3 (Saa3) & NM_011315 & 3.367436 \\
\hline CD53 antigen (Cd53) & NM_007651 & 2.202753 \\
\hline Thrombospondin 1 (Thbs1) & NM_011580 & 2.166568 \\
\hline Lipopolysaccharide binding protein (Lbp) & NM_008489 & 2.148915 \\
\hline Connective tissue growth factor (Ctgf) & NM_010217 & 2.090627 \\
\hline Epiregulin (Ereg) & NM_007950 & 2.087868 \\
\hline Nuclear receptor subfamily 4 , group A, member 1 (Nr4a1) & NM_010444 & 2.059057 \\
\hline CD55 antigen (Cd55) & NM_010016 & 2.0581 \\
\hline Rho-guanine nucleotide exchange factor (Rgnef) & NM_012026 & 2.044236 \\
\hline Retinoic acid receptor responder (tazarotene induced) 1 (Rarres1) & NM_001164763 & 2.041146 \\
\hline Suppressor of cytokine signaling 2 (Socs 2 ), transcript variant 1 & NM_007706 & 2.03834 \\
\hline ATPase, $\mathrm{Na}+/ \mathrm{K}+$ transporting, alpha 2 polypeptide (Atp1a2) & NM_178405 & 2.019371 \\
\hline Adrenomedullin (Adm) & NM_009627 & 2.005821 \\
\hline Acyl-CoA thioesterase 2 (Acot2), nuclear gene encoding mitochondrial protein & NM_134188 & 1.973443 \\
\hline CD34 antigen (Cd34), transcript variant 1 & NM_001111059 & 1.938985 \\
\hline Ets variant gene 4 (E1A enhancer binding protein, E1AF) (Etv4) & NM_008815 & 1.932054 \\
\hline Frizzled homolog 4 (Drosophila) (Fzd4) & NM_008055 & 1.91573 \\
\hline Creatine kinase, mitochondrial 1, ubiquitous ( $\mathrm{Ckmt} 1)$, nuclear gene encoding mitochondrial protein & NM_009897 & 1.912341 \\
\hline Cyclin D1 (Ccnd1) & NM_007631 & 1.910614 \\
\hline Kruppel-like factor 4 (gut) (Klf4) & NM_010637 & 1.893031 \\
\hline Fibroblast growth factor receptor 3 (Fgfr3), transcript variant 1 & NM_008010 & 1.863915 \\
\hline Inhibitor of DNA binding $2(\operatorname{Id} 2)$ & NM_010496 & 1.861815 \\
\hline Dual specificity phosphatase 6 (Dusp6) & NM_026268 & 1.844081 \\
\hline Acid phosphatase 5, tartrate resistant (Acp5), transcript variant 2 & NM_001102404 & 1.842465 \\
\hline Oxidized low density lipoprotein (lectin-like) receptor 1 (Olr1) & NM_138648 & 1.810867 \\
\hline Suppressor of variegation 3-9 homolog 1 (Drosophila) (Suv39h1) & NM_011514 & 1.802061 \\
\hline Cell division cycle 6 (Cdc6), transcript variant 1 & NM_011799 & 1.768399 \\
\hline Phosphodiesterase 4B, cAMP specific (Pde4b), transcript variant 1 & NM_019840 & 1.768212 \\
\hline Serum amyloid A 1 (Saa1) & NM_009117 & 1.762458 \\
\hline Matrix metallopeptidase $1 \mathrm{~b}$ (interstitial collagenase) (Mmp1b) & NM_032007 & 1.761906 \\
\hline Proviral integration site 1 (Pim1) & NM_008842 & 1.741448 \\
\hline
\end{tabular}

differentiation of 3T3-L1 cells, suggest that miR-27b is involved in the regulation of adipocyte differentiation- or lipid metabolism-related gene expression.

3.2. Identification of the MiR-27b-3p-Regulated Genes Responsible for Adipocyte Differentiation and Lipid Metabolism. To determine the target genes of miR-27b-3p in 3T3-L1 cells during adipocyte differentiation, we carried out comprehensive DNA microarray analysis that compared the gene expression patterns of differentiated 3T3-L1 cells transfected with either miR-27b-3p or control miR. Of note, miR-27b-3p significantly up-regulated adipocyte differentiation- and lipid metabolism-related genes such as Acyl-CoA thioesterase 2 (ACOT2), prolactin family 2, subfamily c, member 5 (PRL2C5), epiregulin (EREG), cyclin D1 (CCND1) and krüppel-like transcription factor 4 (KLF4) (Table 1). In contrast, miR-27b-3p down-regulated aldoketo reductase family 1, member B7 (AKR1B7), glutathione S-transferase, alpha 4 (GSTA4), endothelin receptor type A (EDNRA), and signal transducer and activator of transcription 1 (STAT1) (Table 2). GO enrichment analysis of the microarray results revealed a general trend showing that miR-27b-3p regulated a group of genes during the biological regulation and cellular process (Figure 2). In the category of cellular process, we identified cellular metabolic process-related genes including interleukin-1 $\beta$ (NM_008361), C-C motif chemokine 5 (NM_013653), ACOT2 (NM_134188), suppressor of cytokine signaling 2 (NM_007706). Notably, although the basal signal intensity of ACOT2 was higher than the other genes, the altered ratio of ACOT2 mRNA was remarkedly larger. To validate ACOT2 as a responsive gene of miR-27b-3p, we confirmed the increased ACOT2 protein expression in miR-27b-3ptransfected 3T3-L1 cells after differentiation (Figure 3). In summary, we focused on ACOT2, which is known as an enzyme that facilitates the hydrolysis of CoA and free fatty acids.

\subsection{ACOT2 Contributes to MiR-27b-Driven Adipocyte} Differentiation. Next, we designed an ACOT2-specific siRNA for the knockdown of ACOT2 expression. Treatment with ACOT2-specific siRNAs successfully suppressed expression of ACOT2 protein (Figure 4(a)). We examined the impact of 
TABLE 2: Microarray analysis of gene expression decreases on miR-27b transfection.

\begin{tabular}{lcc}
\hline Gene description & Gene accession & Fold increase \\
\hline Natriuretic peptide receptor 1 (Npr1) & NM_008727 & 0.57428 \\
Dickkopf homolog 2 (Xenopus laevis) (Dkk2) & NM_020265 & 0.572471 \\
Aldo-keto reductase family 1, member B7 (Akr1b7) & NM_009731 & NM_010357 \\
Glutathione S-transferase, alpha 4 (Gsta4) & NM_008361 & 0.553844 \\
Interleukin 1 beta (Il1b) & NM_029662 & 0.551271 \\
Major facilitator superfamily domain containing 2A (Mfsd2a) & NM_008153 & 0.543772 \\
Chemokine-like receptor 1 (Cmklr1) & NM_001205349 \\
Lipase, family member K (Lipk), transcript variant 1 & NM_029495 \\
Epithelial stromal interaction 1 (breast) (Epsti1), transcript variant a & NM_010332 & 0.541809 \\
Endothelin receptor type A (Ednra) & NM_013653 & 0.530848 \\
Chemokine (C-C motif) ligand 5 (Ccl5) & NM_009283 \\
Signal transducer and activator of transcription 1 (Stat1), transcript variant 2 & NM_008532 \\
Epithelial cell adhesion molecule (Epcam) & NM_001077353 & 0.530183 \\
Glutathione S-transferase, alpha 3 (Gsta3), transcript variant 1 & NM_009144 \\
Secreted frizzled-related protein 2 (Sfrp2) & 0.514737 \\
\hline
\end{tabular}

ACOT2 on lipid accumulation and adipocyte differentiation. ACOT2-specific siRNAs significantly reduced lipid accumulation, as well as the amount of TG contents in 3T3-L1 cells after differentiation compared to negative control siRNAs (Figures 4(b) and 4(c)). Furthermore, in order to investigate whether miR-27b-3p-mediated the up-regulation of ACOT2 to promote adipocyte differentiation, we transfected 3T3-L1 cells with both miR-27b-3p and ACOT2-siRNA and then evaluated adipocyte differentiation. As shown in Figure 5, the combined treatment of miR-27b with the ACOT2-siRNA group significantly reduced TG contents in 3T3-L1 cells after differentiation compared to that treated with miR-27b-3p alone. In addition, ACOT2-siRNA cancelled the increase in TG contents induced by miR-27b-3p treatment and recovered TG contents to the same level as the control group. These results clearly demonstrate that ACOT2 predominantly contributes to the promotion of adipocyte differentiation by up-regulating miR-27b.

\section{Discussion}

Several studies have indicated that miR-27b plays an important role in lipid metabolism and adipogenesis $[24,25]$. It has been reported that miR-27b is down-regulated in in vitro cultured adipocytes and adipose tissue in obesity-related models and that miR-27b impairs adipocyte differentiation in 3T3-L1 cells and human adipose-derived mesenchymal stem cells via its associations with PPAR $\gamma$ and $\mathrm{C} / \mathrm{EBP} \alpha$ [24-27]. In zebrafish, depletion of miR-27b has shown increases in $\operatorname{PPAR} \gamma, \mathrm{C} / \mathrm{EBP} \alpha$, and SREBP-1c, resulting in the enhancement of adipocyte hyperplasia [28]. In addition to these regulators, $\mathrm{miR}-27 \mathrm{~b}$ negatively regulates lysyl oxidase and other genes involved in adipocyte differentiation [29]. Thus, these studies suggest that miR-27b negatively regulates NAFLD pathogenesis. Surprisingly, we have nevertheless found that miR-27b was upregulated in NAFLD biopsy samples from both early and late stages of NASH. In agreement with our own results, an increase in miR-27b was observed in fat tissue and under hypoxic conditions [25]. In summary, alterations in miR-27b expression in NAFLD remain controversial.

In addition to miR-27b, another miR-27 family gene miR$27 \mathrm{a}$ has been known as a negative regulator of adipogenesis. The expression of miR-27a was down-regulated upon adipogenic differentiation of 3T3-L1 preadipocytes. Moreover, miR27a suppressed lipid accumulation in rat hepatic stellate cells and human hepatoma cells by targeting retinoid $\mathrm{X}$ receptor $\alpha$ $[30,31]$ and impair adipocyte differentiation by targeting PPAR $\gamma$ [32]. Together, these finding suggest a potential role of miR-27a in multiple metabolic pathways during NAFLD pathogenesis. However, our miRNA microarray data showed up-regulation of miR-27a as well as miR-27b. Although the ratio of hold increased of $\mathrm{miR}-27 \mathrm{a}$ was smaller than that of miR-27b, the up-regulation of miR-27a was observed in from onset of NAFLD to late stages of NASH. Several discrepancies about specific miRNA expression in NAFLD has been reported. The mechanisms remain unclear, but it is thought that the co-regulatory networks of noncoding RNAs including miRNAs, long noncoding RNAs, circular RNAs, P-element-induced wimpy testis (PIWI)-interacting RNAs, and enhancer RNAs may be able to explain the discrepancies [33]. As current reports suggested the potential synergistic effect of co-regulation and interactions between these noncoding RNAs, the co-regulatory network may expand the understanding of the molecular regulation and its complexity in NAFLD development and progression [34].

Several pathways by which the amount of miRNA in a tissue is increased have been proposed. In addition to the biosynthesis of miRNA in cells, recent studies have indicated the existence of circulating miRNAs and the intercellular transfer of miRNAs from distinct donor cells to recipient cells via blood circulation. Exosomes are a known miRNA supply source from donor cells. Exosomes are nano-scale extracellular vehicles (EVs) released by various types of cells [35]. EVs contain a variety of cargos including proteins, mRNAs and miRNAs in donor cells, and are involved in the cell-cell communications with recipient target cells by transferring them [36]. Notably, this paracrine signaling of miRNA plays an important 


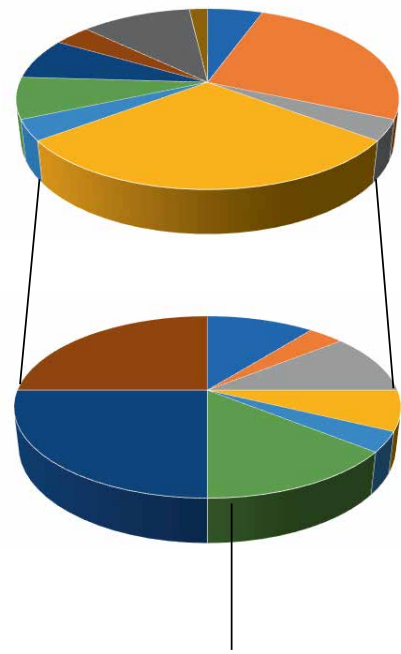

\begin{tabular}{lc} 
GO term (biological process) & Count \\
\hline Biological adhesion (GO:0022610) & 3 \\
Biological regulation (GO:0065007) & 14 \\
Cell proliferation (GO:0008283) & 2 \\
Cellular process (GO:0009987) & 16 \\
Immune system process (GO:0002376) & 2 \\
\hline Localization (GO:0051179) & 4 \\
Metabolic process (GO:0008152) & 4 \\
Multicellular organismal process (GO:0032501) & 2 \\
Response to stimulus (GO:0050896) & 6 \\
Rhythmic process (GO:0048511) & 1 \\
\hline GO term (cellular process) & Count \\
\hline Cell communication (GO:0007154) & 3 \\
Cell cycle checkpoint (GO:0000075) & 1 \\
Cell cycle (GO:0007049) & 3 \\
Cell death (GO:0008219) & 2 \\
Cellular developmental process (GO:0048869) & 1 \\
Cellular metabolic process (GO:0044237) & 4 \\
Cellular response to stimulus (GO:0051716) & 7 \\
Signal transduction (GO:0007165) & 7 \\
\hline
\end{tabular}

Interleukin-1 beta (NM_008361)

C-C motif chemokine 5 (NM 013653)

Acyl-coenzyme A thioesterase 2 (NM_134188)

Suppressor of cytokine signaling 2 (NM_007706)

FIGURE 2: Functional GO enrichment analysis showed a significant phenotypic difference in differentiated 3T3-L1 cells after miR-27b-3p transfection. The microarray data was assessed for biological process function using PANTHER. Enriched biological functions (biological process) of selected up-regulated and down-regulated genes in Tables 1 and 2 (upper pie chart) and the gene counts involved in each process were shown. Subcategories for cellular process were shown in lower pie chart.

role in cancer metastasis [37]. In this line, increases of miR-27b in the liver tissue of NAFLD patients might be explained by both biogenesis in hepatic cells and paracrine regulation. In our previous study, miR-27b was found to be up-regulated in both liver tissue and serum in patients. These suggested the possibility that miR-27b in EVs is provided by distant donor cells and accumulates in the liver tissue of NAFLD patients. This pathway might explain the up-regulation of miR-27b in NAFLD patients.

In this study, we performed DNA microarray analysis and identified up-regulated and down-regulated mRNA via the transfection of miR-27b-3p in 3T3-L1 cells. However, PPAR $\gamma$, C/EBP $\alpha$, and SREBP-1c mRNAs did not exhibit significant changes in our microarray data. GO analysis was utilized to identify the putative candidate genes of miR-27b-3p implicated in lipid metabolism and adipocyte differentiation. Among genes altered by miR-27b-3p treatment, we focused on ACOT2, which is an enzyme of the ACOT family involved in the hydrolysis of fatty acyl-CoAs into free fatty acids and CoA-SH $[38,39]$. ACOT2 is localized to the mitochondrial matrix and maintains an adequate rate of $\beta$-oxidation by retaining the same level of coenzymes used in the tricarboxylic acid (TCA) cycle and in $\beta$-oxidation itself. ACOT2 expression in the mitochondria increased following adipocyte differentiation $[38,39]$. In this study, we have shown that miR-27b increased the expression of ACOT2, and that the increase in ACOT2 played an important role in lipid accumulation and preadipocyte differentiation. Moreover, knockdown of ACOT2 mRNA and protein by a specific siRNA clearly showed the

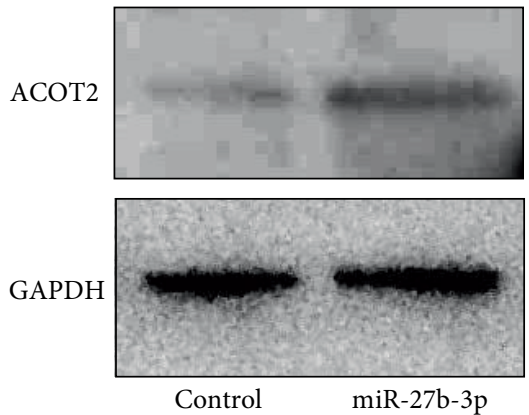

Figure 3: MiR-27b induces ACOT2 expression during adipocyte differentiation. Immunoblotting of ACOT2 in control-miR and miR27b transfected 3T3-L1 cells after adipocyte differentiation. GAPDH was used as an internal standard. Representative data from three different images are shown.

suppression of adipocyte differentiation. These results therefore plainly indicate that the miR-27b-ACOT2 axis promotes lipid accumulation and adipocyte differentiation, process which result in NAFLD pathogenesis.

Several studies have shown that miR-27b regulates not only adipogenesis, but also cell proliferation, angiogenesis, and tumor development $[14,15,40,41]$. Nonetheless, the role of miR-27b has not been fully elucidated. For example, upregulated miR-27b in the serum of Kawasaki disease patients enhanced endothelial cell proliferation and migration by targeting Smad7 [40]. In contrast, down-regulated miR-27b promoted the activation of adenosine monophosphate-activated 


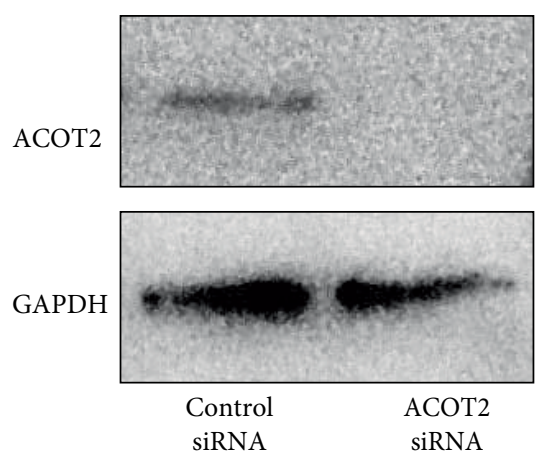

(a)

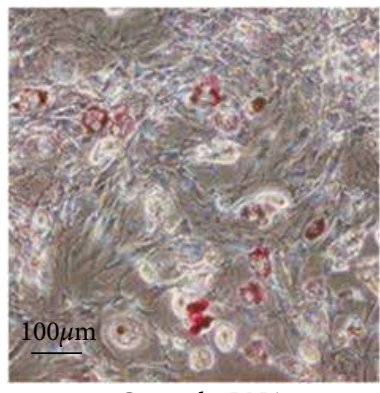

Control siRNA

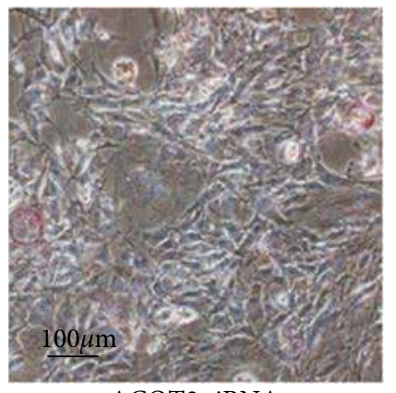

ACOT2 siRNA

(b)

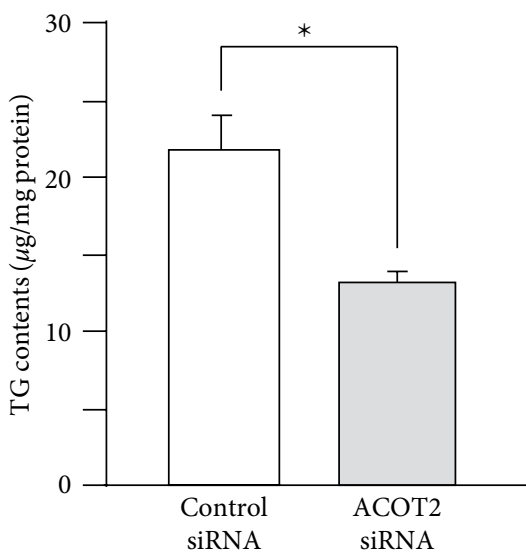

(c)
FIGURE 4: Knockdown of ACOT2 expression suppresses adipocyte differentiation. (a) Immunoblotting of ACOT2 in control-siRNA and ACOT2-siRNA transfected 3T3-L1 cells after adipocyte differentiation. Representative data from three different images are shown. (b) Oil red O staining of control siRNA and ACOT2 siRNA transfected 3T3-L1 cells after adipocyte differentiation. Representative data from three different images are shown. Scale bars: $100 \mu \mathrm{m}$. (c) Intracellularly TG contents of control siRNA and ACOT2 siRNA transfected 3T3-L1 cells were measured. Each column represents the mean \pm SEM from 6 independent experiments. ${ }^{*}$ Significantly difference than control siRNA by Student's $t$-test $(P<0.05)$.

protein kinase, which induced angiogenic tube formation and the migration of endothelial cells [15]. In addition, miR-27b highly expressed in sheep skeletal muscle directly reduced myostatin mRNA and led to cell proliferation [41]. In contrast, down-regulated miR-27b in oral lichen planus promotes human oral keratinocytes proliferation via increased Polo-like Kinase-2 expression [14]. Moreover, the down-regulation and

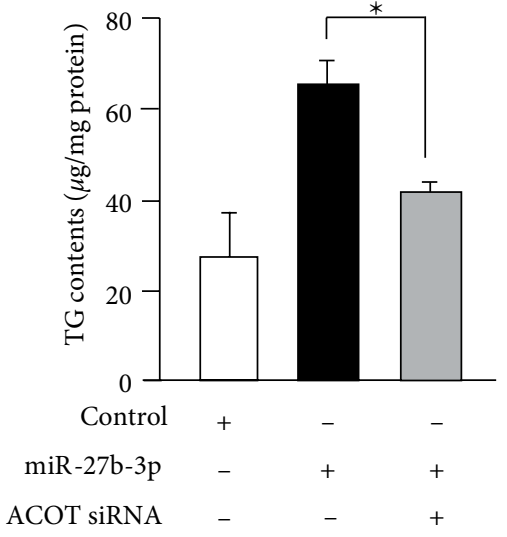

FIGURE 5: ACOT2 knockdown cancelled miR-27b-driven adipocyte differentiation. Intracellular TG contents of 3T3-L1 cells treated with a combination of miR-27b and ACOT2-siRNA were measured. Each column represents the mean \pm SEM from 5 to 7 independent experiments. ${ }^{*}$ Significantly difference than the combination of miR-27b and ACOT2siRNA group by Scheffe's method after ANOVA $(P<0.05)$.

up-regulation of miR-27b promotes breast cancer metastasis and gastric cancer, respectively. These studies suggest that miR-27b has different expression pattern and functions depending on the cell type, biological process, diseases, and experimental conditions.

Our study provides the first evidence that up-regulated miR-27b in NAFLD patients promotes adipogenesis and lipid deposition in 3T3-L1 cells during adipocyte differentiation. A similar experiment showed inconsistent results; the authors found that miR-27b in 3T3-L1 cells stimulated by DEX, IBMX, and INS became down-regulated and that exogenous miR-27b reduced lipid accumulation [25]. In our model, we stimulated 3T3-L1 cells with DEX, IBMX, INS, and ROSI to achieve adipocyte differentiation. This occurred in response to NAFLD development under these signaling cascades. Notably, PPAR $\gamma$ is a master transcriptional regulator of adipogenesis and lipid metabolism [42]. Indeed, since increased PPAR $\gamma$ expression has been found in steatotic livers, it has been suggested that the role of PPAR $\gamma$ in the activation of lipogenic genes may contribute to the development of steatosis [1]. In agreement with PPAR $\gamma$ activation in the fatty liver of obese human subjects, we stimulated cells with PPAR $\gamma$ and other inducers, which resulted in the enhancement of adipocyte differentiation [19]. Thus, PPAR $\gamma$ activation might be critical for miR-27b expression under PPAR $\gamma$ signaling; however, further studies are required for elucidation.

$\operatorname{PPAR} \gamma$ is highly expressed not only in adipose tissue, but also in the liver of NAFLD patients and in experimental models $[43,44]$. PPAR $\gamma$ deletion in mouse hepatocytes has been shown to protect against the development of steatosis [45]. Moreover, several studies have shown that PPAR $\gamma$ activation can prevent the progression of hepatic steatosis in murine models [46], and that treatment with the PPAR $y$ agonist rosiglitazone exerts similar effects [47]. The protective effects of PPAR $y$ could be the result of higher insulin 
sensitivity in adipose tissue and skeletal muscles, leading to a reduction in free fatty acid deposition in the liver [45]. PPAR $y$ has been shown to induce adiponectin, which also contributes to insulin sensitivity, and PPAR $\alpha$ expression, in turn leading to further hepatic fatty acid oxidation. Furthermore, PPAR $\gamma$ expression has been shown to promote anti-inflammatory and anti-fibrotic effects in stellate cells, macrophages, and epithelial cells [48]. In addition to adipocyte differentiation, it remains unclear whether miR-27b is involved in such PPAR $\gamma$-related effects as improvements in insulin sensitivity, antiinflammatory and anti-fibrotic effects.

\section{Conclusions}

In summary, this study has successfully provided important information about the roles of up-regulated miR-27b in the context of NAFLD pathogenesis. Our result suggests that miR27b-3p induces ACOT2 expression and that the miR-27b-3pACOT2 axis contributes to adipocyte differentiation. Further investigation will be required to clarify the exact mechanisms at work. Nonetheless, miR-27b-ACOT2 axis may potentially play a role in the pathological progression of NAFLD.

\section{Data Availability}

All data used to support the findings of this study are included within the article.

\section{Conflicts of Interest}

The authors declare that they have no conflicts of interest.

\section{Acknowledgments}

We thank Tomomi Niibayashi (Shimane University), and Kazi Hossain Helal (Shimane University) for their experimental assistance. This work was supported by JSPS KAKENHI (Grants-in-Aid for Scientific Research) [grant numbers 15H04757, 19H04032].

\section{References}

[1] A. Wieckowska, B. G. Papouchado, Z. Li, R. Lopez, N. N. Zein, and A. E. Feldstein, "Increased hepatic and circulating interleukin-6 levels in human nonalcoholic steatohepatitis," The American Journal of Gastroenterology, vol. 103, no. 6, pp. 1372-1379, 2008.

[2] L. A. Adams, O. R. Waters, M. W. Knuiman, R. R. Elliott, and J. K. Olynyk, "NAFLD as a risk factor for the development of diabetes and the metabolic syndrome: an eleven-year followup study," The American Journal of Gastroenterology, vol. 104, no. 4, pp. 861-867, 2009.

[3] M. Ekstedt, L. E. Franzen, U. L. Mathiesen et al., "Long-term follow-up of patients with NAFLD and elevated liver enzymes," Hepatology, vol. 44, no. 4, pp. 865-873, 2006.

[4] A. Berlanga, E. Guiu-Jurado, J. A. Porras, and T. Auguet, "Molecular pathways in non-alcoholic fatty liver disease," Clinical and Experimental Gastroenterology, pp. 221-239, 2014.
[5] G. Baffy, "MicroRNAs in nonalcoholic fatty liver disease," Journal of Clinical Medicine, vol. 4, no. 12, pp. 1977-1988, 2015.

[6] R. C. Lee, R. L. Feinbaum, and V. Ambros, "The C. elegans heterochronic gene lin-4 encodes small RNAs with antisense complementarity to lin-14," Cell, vol. 75, no. 5, pp. 843-854, 1993.

[7] B. J. Reinhart, F. J. Slack, M. Basson et al., "The 21-nucleotide let-7 RNA regulates developmental timing in Caenorhabditis elegans," Nature, vol. 403, no. 6772, pp. 901-906, 2000.

[8] D. P. Bartel, "MicroRNAs: genomics, biogenesis, mechanism, and function," Cell, vol. 116, no. 2, pp. 281-297, 2004.

[9] O. Cheung, P. Puri, C. Eicken et al., "Nonalcoholic steatohepatitis is associated with altered hepatic MicroRNA expression," Hepatology, vol. 48, no. 6, pp. 1810-1820, 2008.

[10] P. P. Becker, M. Rau, J. Schmitt et al., "Performance of serum microRNAs-122, -192 and -21 as biomarkers in patients with non-alcoholic steatohepatitis," PLoS One, vol. 10, no. 11, p. e0142661, 2015.

[11] S. H. Hsu, B. Wang, J. Kota et al., "Essential metabolic, antiinflammatory, and anti-tumorigenic functions of miR-122 in liver," Journal of Clinical Investigation, vol. 122, no. 8, pp. 2871-2883, 2012.

[12] T. Kessoku, Y. Honda, Y. Ogawa et al., "Mechanism analysis of microRNA-27b causing the fatty liver formation and insulin resistance at the same onset," Hepatology, vol. 60, p. 746a, 2014.

[13] T. Kessoku, E. Sakai, Y. Honda et al., "Micro Rna-27b causes nonalcoholic steatohepatitis similar to human pathophysiology in murine high-fat diet model," Journal of Hepatology, vol. 60, p. S155, 2014.

[14] J. Chen, G. Du, Y. Chang et al., "Downregulated miR-27b promotes keratinocyte proliferation by targeting PLK2 in oral lichen planus," Journal of Oral Pathology \& Medicine, vol. 48, no. 4, 2019.

[15] Y. Yuan, Z. Zhang, Z. Wang, and J. Liu, "MiRNA-27b regulates angiogenesis by targeting AMPK in mouse ischemic stroke model," Neuroscience, vol. 398, pp. 12-22, 2019.

[16] Y. Xu, Y. F. Han, and B. Ye, "miR-27b-3p is involved in doxorubicin resistance of human anaplastic thyroid cancer cells via targeting peroxisome proliferator-activated receptor gamma," Basic \& Clinical Pharmacology \& Toxicology, vol. 123, no. 6, pp. 670-677, 2018.

[17] J. Jiang, B. Yi, C. Qin, D. Siqing, and C. Wei, "Upregulation of microRNA27b contributes to the migration and invasion of gastric cancer cells via the inhibition of sprouty2mediated ERK signaling," Molecular Medicine Reports, vol. 13, no. 3, pp. 2267-2272, 2016.

[18] X. Chen, Y. Cui, X. Xie, Y. Xing, Z. Yuan, and Y. Wei, "Functional role of miR-27b in the development of gastric cancer," Molecular Medicine Reports, vol. 17, no. 4, pp. 5081-5087, 2018.

[19] K. Katayama, K. Wada, H. Miyoshi et al., "RNA interfering approach for clarifying the PPARgamma pathway using lentiviral vector expressing short hairpin RNA," FEBS Letters, vol. 560, pp. 178-182, 2004.

[20] K. Hirata, K. Wada, Y. Murata, A. Nakajima, T. Yamashiro, and Y. Kamisaki, "Critical role of leukotriene B4 receptor signaling in mouse 3T3-L1 preadipocyte differentiation," Lipids in Health and Disease, vol. 12, p. 122, 2013.

[21] K. Hirata, K. Katayama, A. Nakajima, K. Takada, Y. Kamisaki, and $\mathrm{K}$. Wada, "Role of leukotriene $\mathrm{B}(4)$ receptor signaling in human preadipocyte differentiation," Biochemical and 
Biophysical Research Communications, vol. 429, no. 3-4, pp. 197-203, 2012.

[22] S. M. Niemela, S. Miettinen, Y. Konttinen et al., "Fat tissue," Journal of Craniofacial Surgery, vol. 18, no. 2, pp. 325-335, 2007.

[23] X. Ma, P. Lee, D. J. Chisholm, and D. E. James, "Control of adipocyte differentiation in different fat depots; implications for pathophysiology or therapy," Frontiers in Endocrinology, vol. 6, p. 1, 2015.

[24] K. D. Chen, K. T. Huang, C. C. Lin et al., "MicroRNA-27b enhances the hepatic regenerative properties of adipose-derived mesenchymal stem cells," Molecular Therapy - Nucleic Acids, vol. 5, p. e285, 2016.

[25] Q. Lin, Z. Gao, R. M. Alarcon, J. Ye, and Z. Yun, "A role of miR-27 in the regulation of adipogenesis," FEBS Journal, vol. 276, no. 8, pp. 2348-2358, 2009.

[26] J. Yu, Y. Lv, F. Wang et al., "MiR-27b-3p inhibition enhances browning of epididymal fat in high-fat diet induced obese mice," Frontiers in Endocrinology, vol. 10, p. 38, 2019.

[27] X. Hu, J. Tang, X. Hu et al., "MiR-27b impairs adipocyte differentiation of human adipose tissue-derived mesenchymal stem cells by targeting LPL," Cellular Physiology and Biochemistry, vol. 47, no. 2, pp. 545-555, 2018.

[28] C. C. Hsu, C. Y. Lai, C. Y. Lin, K.-Y. Yeh, and G. Her, "MicroRNA27b depletion enhances endotrophic and intravascular lipid accumulation and induces adipocyte hyperplasia in zebrafish," International Journal of Molecular Sciences, vol. 19, no. 1, p. 93, 2017.

[29] S. Z. Chen, X. Xu, L. F. Ning et al., "miR-27 impairs the adipogenic lineage commitment via targeting lysyl oxidase," Obesity (Silver Spring), vol. 23, no. 12, pp. 2445-2453, 2015.

[30] T. Shirasaki, M. Honda, T. Shimakami et al., "MicroRNA27a regulates lipid metabolism and inhibits hepatitis $\mathrm{C}$ virus replication in human hepatoma cells," Journal of Virology, vol. 87, no. 9, pp. 5270-5286, 2013.

[31] J. Ji, J. Zhang, G. Huang, J. Qian, X. Wang, and S. Mei, “Overexpressed microRNA-27a and $27 \mathrm{~b}$ influence fat accumulation and cell proliferation during rat hepatic stellate cell activation," FEBS Letters, vol. 583, no. 4, pp. 759-766, 2009.

[32] S. Y. Kim, A. Y. Kim, H. W. Lee et al., "miR-27a is a negative regulator of adipocyte differentiation via suppressing PPARgamma expression," Biochemical and Biophysical Research Communications, vol. 392, no. 3, pp. 323-328, 2010.

[33] S. A. Sulaiman, N. I. A. Muhsin, and R. Jamal, "Regulatory non-coding RNAs network in non-alcoholic fatty liver disease," Frontiers in Physiology, vol. 10, p. 279, 2019.

[34] K. Takahashi, I. Yan, H. Haga, and T. Patel, "Long noncoding RNA in liver diseases," Hepatology, vol. 60, no. 2, pp. 744-753, 2014.

[35] M. Tkach and C. Thery, "Communication by extracellular vesicles: where we are and where we need to go," Cell, vol. 164, no. 6, pp. 1226-1232, 2016.

[36] K. Sato, F. Meng, S. Glaser, and G. Alpini, "Exosomes in liver pathology," Journal of Hepatology, vol. 65, no. 1, pp. 213-221, 2016.

[37] A. Hoshino, B. Costa-Silva, T. L. Shen et al., "Tumour exosome integrins determine organotropic metastasis," Nature, vol. 527, no. 7578, pp. 329-335, 2015.
[38] C. Moffat, L. Bhatia, T. Nguyen et al., "Acyl-CoA thioesterase-2 facilitates mitochondrial fatty acid oxidation in the liver," The Journal of Lipid Research, vol. 55, no. 12, pp. 2458-2470, 2014.

[39] A. Momose, M. Fujita, T. Ohtomo et al., "Regulated expression of acyl-CoA thioesterases in the differentiation of cultured rat brown adipocytes," Biochemical and Biophysical Research Communications, vol. 404, no. 1, pp. 74-78, 2011.

[40] X. Rong, D. Ge, D. Shen et al., "miR-27b suppresses endothelial cell proliferation and migration by targeting Smad7 in Kawasaki disease," Cellular Physiology and Biochemistry, vol. 48, pp. 1804-1814, 2018.

[41] W. Zhang, S. Y. Wang, S. Y. Deng et al., "MiR-27b promotes sheep skeletal muscle satellite cell proliferation by targeting myostatin gene," Journal of Genetics, vol. 97, no. 5, pp. 1107-1117, 2018.

[42] M. Okamura, T. Inagaki, T. Tanaka, and J. Sakai, "Role of histone methylation and demethylation in adipogenesis and obesity," Organogenesis, vol. 6, no. 1, pp. 24-32, 2010.

[43] S. Yu, K. Matsusue, P. Kashireddy et al., "Adipocyte-specific gene expression and adipogenic steatosis in the mouse liver due to peroxisome proliferator-activated receptor gammal (PPARgamma1) overexpression," The Journal of Biological Chemistry, vol. 278, no. 1, pp. 498-505, 2003.

[44] P. Pettinelli and L. A. Videla, "Up-regulation of PPAR-gamma mRNA expression in the liver of obese patients: an additional reinforcing lipogenic mechanism to SREBP-1c induction," The Journal of Clinical Endocrinology \& Metabolism, vol. 96, no. 5, pp. 1424-1430, 2011.

[45] K. Matsusue, M. Haluzik, G. Lambert et al., "Liver-specific disruption of PPARgamma in leptin-deficient mice improves fatty liver but aggravates diabetic phenotypes," Journal of Clinical Investigation, vol. 111, no. 5, pp. 737-747, 2003.

[46] N. Maeda, M. Takahashi, T. Funahashi et al., "PPARgamma ligands increase expression and plasma concentrations of adiponectin, an adipose-derived protein," Diabetes, vol. 50, no. 9, pp. 2094-2099, 2001.

[47] A. A. Gupte, J. Z. Liu, Y. Ren et al., "Rosiglitazone attenuates age- and diet-associated nonalcoholic steatohepatitis in male low-density lipoprotein receptor knockout mice," Hepatology, vol. 52, no. 6, pp. 2001-2011, 2010.

[48] A. Vetuschi, S. Pompili, E. Gaudio, G. Latella, and R. Sferra, "PPAR-gamma with its anti-inflammatory and anti-fibrotic action could be an effective therapeutic target in IBD," European Review for Medical and Pharmacological Sciences, vol. 22, no. 24, pp. 8839-8848, 2018. 


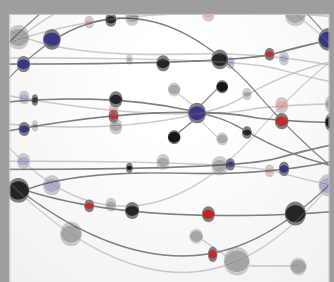

The Scientific World Journal
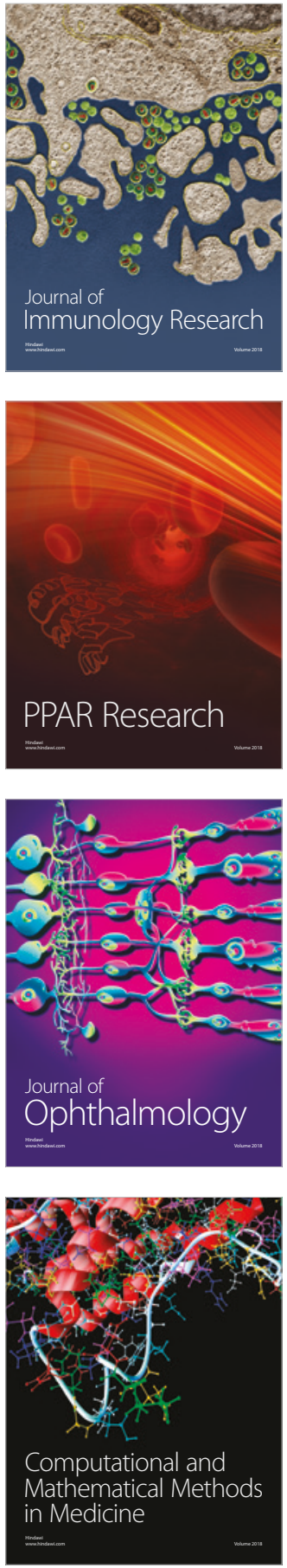

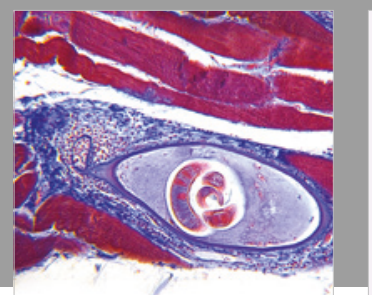

Gastroenterology Research and Practice

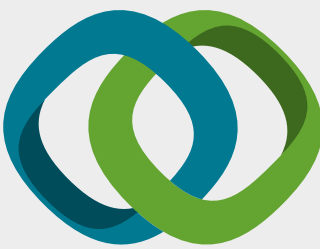

\section{Hindawi}

Submit your manuscripts at

www.hindawi.com
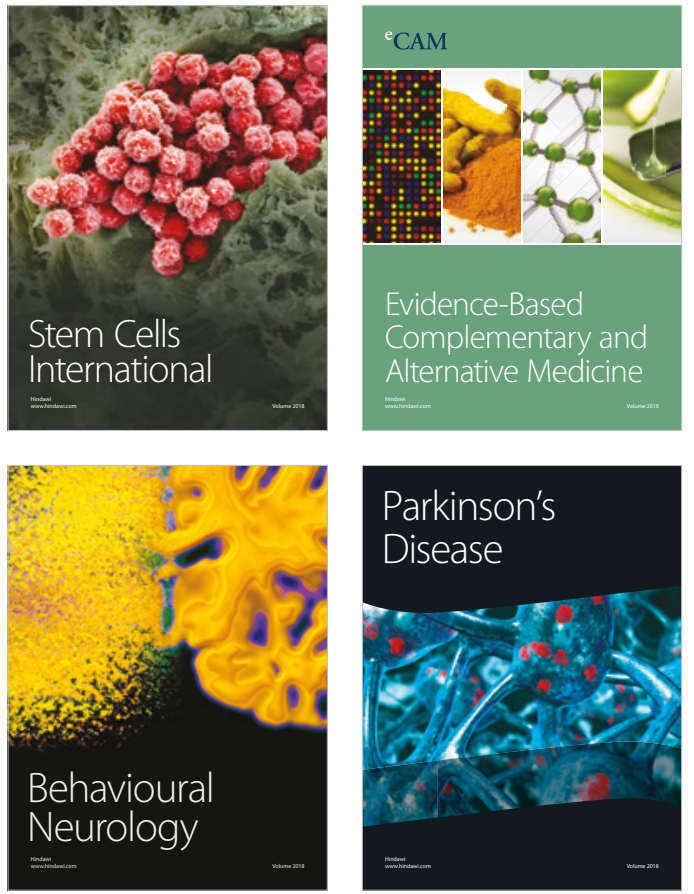

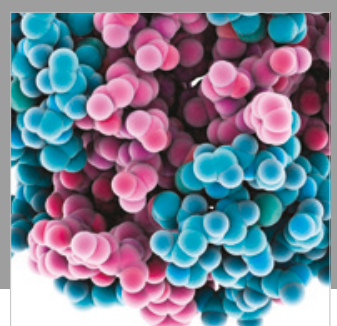

ournal of

Diabetes Research

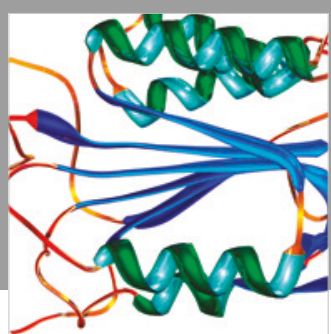

Disease Markers
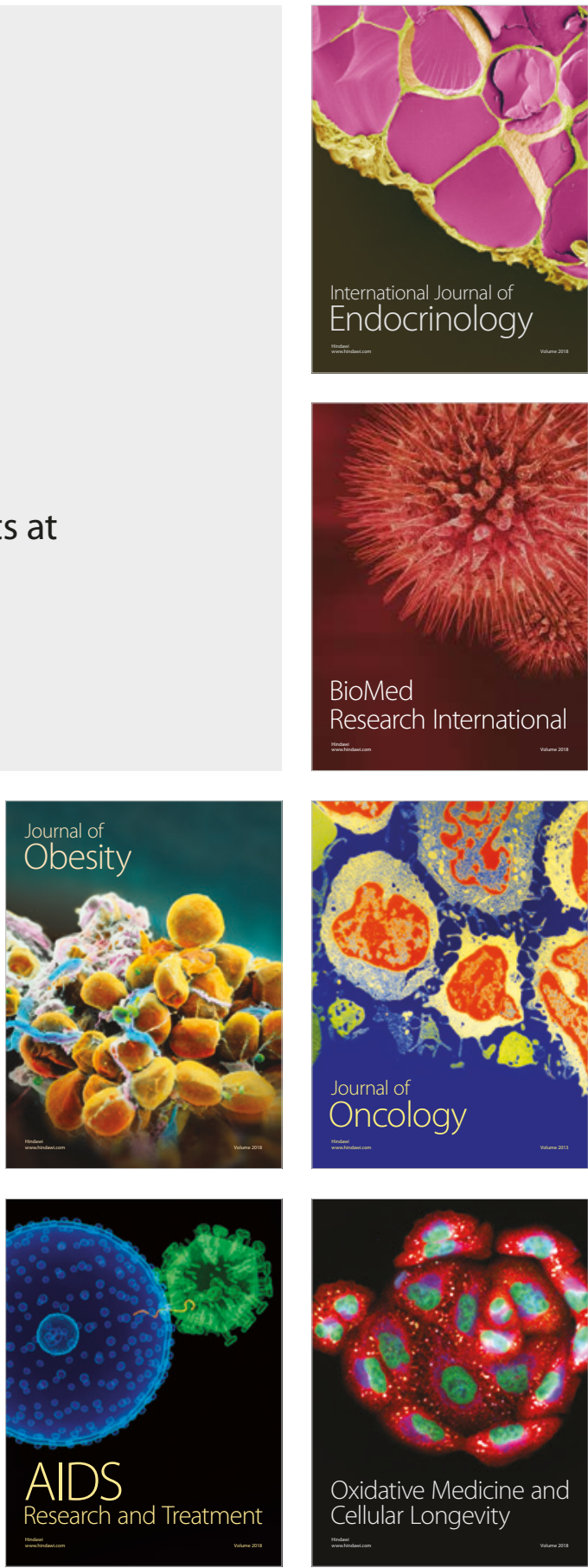